\title{
CHARACTERIZATIONS OF A NEW CORDYCEPS CICADAE ISOLATE AND PRODUCTION OF ADENOSINE AND CORDYCEPIN
}

\begin{abstract}
Yongjun Wang ${ }^{*}$, Yanbin Guo ${ }^{2}$, Liqin Zhang ${ }^{1}$, Jia Wu ${ }^{1}$
${ }^{1}$ Key Laboratory of Forest Protection, College of Forestry and Biotechnology, Zhejiang Agricultural and Forestry University, Lin'an 311300 China; ${ }^{2}$ College of Resources and Environmental Sciences, China Agricultural University, Beijing 100094 China.

Submitted: November 30, 2010; Approved: January 16, 2012.

ABSTRACT

Cordyceps is a fastidious pathogenic fungus infecting insects, and recent years have witnessed rapid progress in its medical properties. In this study, a wild isolate, C. cicadae MP12, was characterized through in vitro cultivation and its nuclear small-subunit (SSU) ribosomal DNA (rDNA) data. In vitro culture of $C$. cicadae MP12 was established by growing its fruiting bodies in a solid matrix. C. cicadae MP12 was inoculated into Cryptotympana atrata cicada pupae for in vivo culture, where the fungi developed its fruiting body as well. The contents of adenosine and cordycepin in dried fruiting bodies after culture were $1421.45 \mu \mathrm{g} / \mathrm{g}$ and $1398.12 \mu \mathrm{g} / \mathrm{g}$, respectively. Therefore, the established cultures from this study could be used for the production of various medically important metabolic substances.
\end{abstract}

Key words: Cordyceps cicadae, cultivation, adenosine, cordycepin.

\section{INTRODUCTION}

Cordyceps is one of the entomopathogenic fungi capable of invading a specific insect and kills the host eventually. After hibernating the winter inside the host, it forms a fruiting body on the surface of the insect's cadaver during the summer. $C$. sinensis, is the most popular Cordyceps, which is a parasite of the Hepialus armoricanus larvae. The fungus has long been used to promote longevity, relieve exhaustion and treat numerous diseases in Chinese traditional medicines (19). Recent studies have demonstrated that various species in this genus possess multiple pharmacological properties, including anti-tumor, anti-microbial, anti-inflammatory and immunomodulatory effects (5). Furthermore, a variety of effective chemical constituents including cordycepin, adenosine, ergosterol, and myriocin have been isolated from various Cordyceps species $(9,16,26,27)$.

Adenosine is widely distributed in Cordyceps spp. It is also known to have a widespread effect on coronary and cerebral circulation (4), control of blood flow (1), prevention of cardiac arrhythmias (18). Cordycepin (3'-deoxyadenosine) is a nucleoside analogue, which exhibits a broad spectrum of biological activity $(2,7)$. Cordycepin can be converted into its 5'-mono, di and triphosphates intracellularly to inhibit the activity of ribose-phosphate pyrophosphokinase and 5'phosphoribosyl- 1'- pyrophosphate amidotransferase in the

*Corresponding Author. Mailing address: Key Lab of Forest Protection, College of Forestry and Biotechnology, Zhejiang Agricultural and Forestry University, Lin'an 311300, China.; Tel.: +86 057161069780 Fax: +86 $057163740898 . ;$ E-mail: wangyj@zafu.edu.cn 
de novo biosynthesis of purines and/or the synthesis of nucleic acids, causing the anti-tumor, anti-metastatic and antimicrobial effects $(6,11,13,15,20,22)$. In addition, the antileukemic activity of cordycepin combined with an adenosine deaminase inhibitor and the inhibitory effect of its analogues of 2', 5'-oligoadenylate on human immunodeficiency virus infection have also been reported (8).

A C. militaris-like isolate of C. cicadae MP12 was obtained from the fruiting body forming on the cicada from Hubei province, China. In this study, the biological and morphological characters were described. To assess the potential of $C$. cicadae MP12 as a new fungal resource for medical applications, the in vitro culture methods were established and the contents of adenosine and cordycepin were measured.

\section{MATERIALS AND METHODS}

\section{Microorganisms and insect}

C. cicadae MP12 (its fruiting body looked like $C$. militaris) was obtained from the Shennongjia Mountains, Hubei province, China. Cryptotympana atrata cicada pupae were used as the host for in vivo culture of the fungus in this study.

\section{In vitro culture}

Cultures of $C$. cicadae MP12 were grown on Sabouraud dextrose agar (Difco Laboratories) slants at $25^{\circ} \mathrm{C}$ and maintained on the same medium at 2 to $4^{\circ} \mathrm{C}$. One loopful of spores from 7- to 14-day cultures was used to inoculate a liquid medium consisting of $4 \%$ fructose and $1 \%$ Neopeptone (Difco; $\mathrm{pH}$ adjusted to 7.5 after autoclaving by using sterile $1.0 \mathrm{~N}$ $\mathrm{NaOH}$ ). Each 250-ml flask contained $60 \mathrm{ml}$ of medium, and cultures were incubated at $250 \mathrm{rpm}$ on a rotary shaker for the specified duration.

After 3-days of culture in the flask, $2 \mathrm{ml}$ of mycelia culture was inoculated into a transparent glass bottle containing
RSM medium (rice $40 \mathrm{~g}$, glucose $0.4 \mathrm{~g}$, tryptone $0.2 \mathrm{~g}, \mathrm{MgSO}_{4}$ $0.8 \mathrm{~g}, \mathrm{~K}_{2} \mathrm{HPO}_{4} 0.4 \mathrm{~g}$, vitamin B complex $0.05 \mathrm{~g}, \mathrm{H}_{2} \mathrm{O}_{2} 50 \mathrm{ml}, \mathrm{pH}$ 7.0-7.2; autoclaved for $20 \mathrm{~min}$ at $121^{\circ} \mathrm{C}$ ). The bottle was sealed by a ventilated film after inoculation. The inoculated bottles were incubated in a culture room $\left(25^{\circ} \mathrm{C}, 12 \mathrm{~h}\right)$ for mycelium growth and fruiting body formation.

\section{In vivo culture}

The spores were suspended in phosphate salt buffer $(\mathrm{pH}$ 7.5) after 14-day culture in plates and diluted to $\sim 10^{6}$ spores $/ \mathrm{ml}$. The cicada pupae were collected from nature and surface sterilized using $75 \%(\mathrm{~V} / \mathrm{V})$ ethanol for $5 \mathrm{~min}$. Each pupa was then placed into a transparent glass bottle and sprayed with $1 \mathrm{ml}$ spore suspension. The pupae were incubated in a culture room $\left(25^{\circ} \mathrm{C}, 12 \mathrm{~h}\right)$ for infection to occur.

\section{Conidial measurements}

Between twenty to thirty conidia from 10-20 day old cultures were suspended in $0.01 \%$ Tween 40 and mixed with an equal volume of agarose. Conidia were observed using a Nikon E600 microscope equipped with Nikon DXM 1200 digital camera and Nikon ACT-1 image capture software.

\section{DNA extraction and PCR}

DNA was extracted from the fungal cultures by a modified CTAB method as previously described (21). Approximately 1150 base pairs (bp) of the nuclear smallsubunit ribosomal DNA (SSU rDNA) was amplified using PCR. Amplifications and sequencing reactions were performed as described in Sung et al (21).

\section{Sequence analysis}

Sequences were edited using BioEdit version 7.0.4.1. The programs DNADIST, NEIGHBOR, DNAPARS, SEQBOOT, and CONSENSE, present in the PHYLIP package (Version 3.68) were used to perform the maximum parsimony analyses on the data of SSU rDNA. Ambiguously aligned sequence 
regions were excluded from the data matrix before analysis. Relative support of the resulting trees was determined by 1000 bootstrap replications on informative characters only with the known search options (3). The phylogenetic tree was generated using TreeView version 1.6.

\section{Extraction and analysis of adenosine and cordycepin}

The fruiting bodies and mycelia of $C$. cicadae MP12 were kept in a vacuum incubator at $50^{\circ} \mathrm{C}$ overnight for drying. The samples were ground and extracted in deionized water.

Chromatographic separations were performed using a Waters HPLC system (Millipore, Waters Division, Milford, MA). The HPLC column was a C8 reversed-phase column for nucleotides (YMC-Pack FA), which was packed with Si 60 (particle size $5 \mu \mathrm{m}, 250-4.6 \mathrm{~mm}$ ). Analysis of the chromatograms was performed using Waters Millennium software. Extractions of C. cicadae MP12 fruiting body were routinely monitored at a wavelength of $258 \mathrm{~nm}$. Peak area measurements were used to quantitate retinoid amounts using a standard curve based on cordycepin (Sigma-Aldrich, St. Louis, MO) and adenosine standards (Sigma-Aldrich, St. Louis, MO) and normalized using an internal standard. The solvent system for elution from the YMC-Pack FA column consisted of methyl alcohol:Milli-Q water (85:15, by vol). Separations were made at a flow rate of $0.7 \mathrm{ml} / \mathrm{min}$. The column temperature was kept constant at $25^{\circ} \mathrm{C}$. The retention time (RT, in min) on the YMC-Pack FA column ranged from 1 to $18 \mathrm{~min}$.

\section{Data analysis}

Statistical significance between the groups was determined by paired t-test and one-way ANOVA for repeated measures. Results with $p<0.05$ were considered statistically significant. Data were assessed using the SPSS software (version 15.0, SPSS Inc., Chicago, Illinois).

\section{RESULTS}

\section{Cultivation of C. cicadae MP12}

After storage of the fungus in the Sabouraud dextrose agar plate for 7 days (Fig. 1), C. cicadae MP12 spores were collected and suspended in phosphate salt buffer $(\mathrm{pH} 7.8)$ and diluted to $10^{8} \mathrm{spores} / \mathrm{ml}$. The suspensions were inoculated into the RSM medium to induce sexual phase. The fruiting bodies formed after 14 days since inoculation and reached at $\sim 8 \mathrm{~cm}$ in length and $\sim 0.8 \mathrm{~cm}$ in width 29 days later (Fig. 2A). The fruiting bodies were bright orange on the outside and milky inside. The average weight of the dried fruiting body was about 8.91g (statistical data not shown).

About two hundred cicada pupae were used for in vivo cultivation of the fungi. The pupae were soaked in spore suspension for $30 \mathrm{~min}$. The cicada died 6 days after inoculation. $95 \%$ of insects were inoculated successfully. The fruiting bodies started to grow on the insect surface 16 days since cicada died and reached at $\sim 8 \mathrm{~cm}$ in length and $\sim 0.8 \mathrm{~cm}$ in width 29 days later (Fig 2C, D). Bright orange fruiting bodies were observed. Figure 2D illustrated the fruiting body of $C$. cicadae MP12 grown on a cicada after metamorphosis.
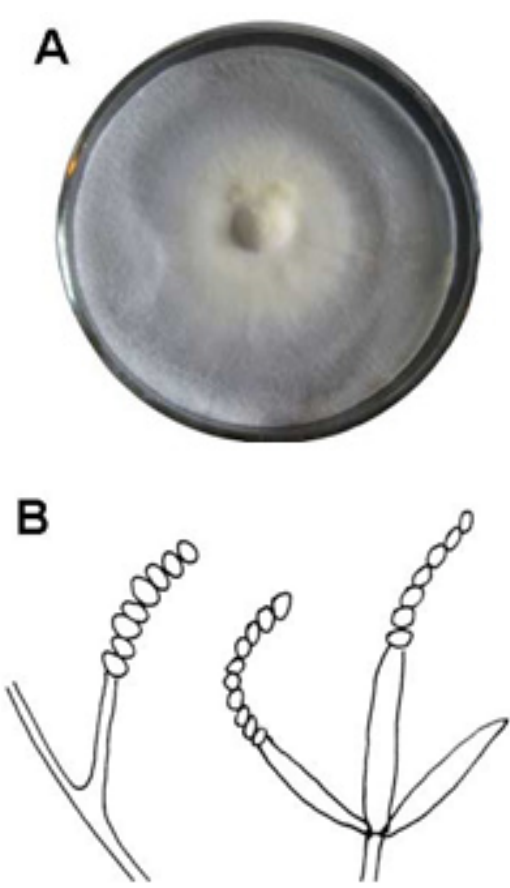

Figure 1. The colony of $C$. cicadae MP12 growing on the Sabouraud dextrose agar plate (A) and line drawings of hyphae and conidia (B) (Scale bar: $10 \mu \mathrm{m}$ ). 
A
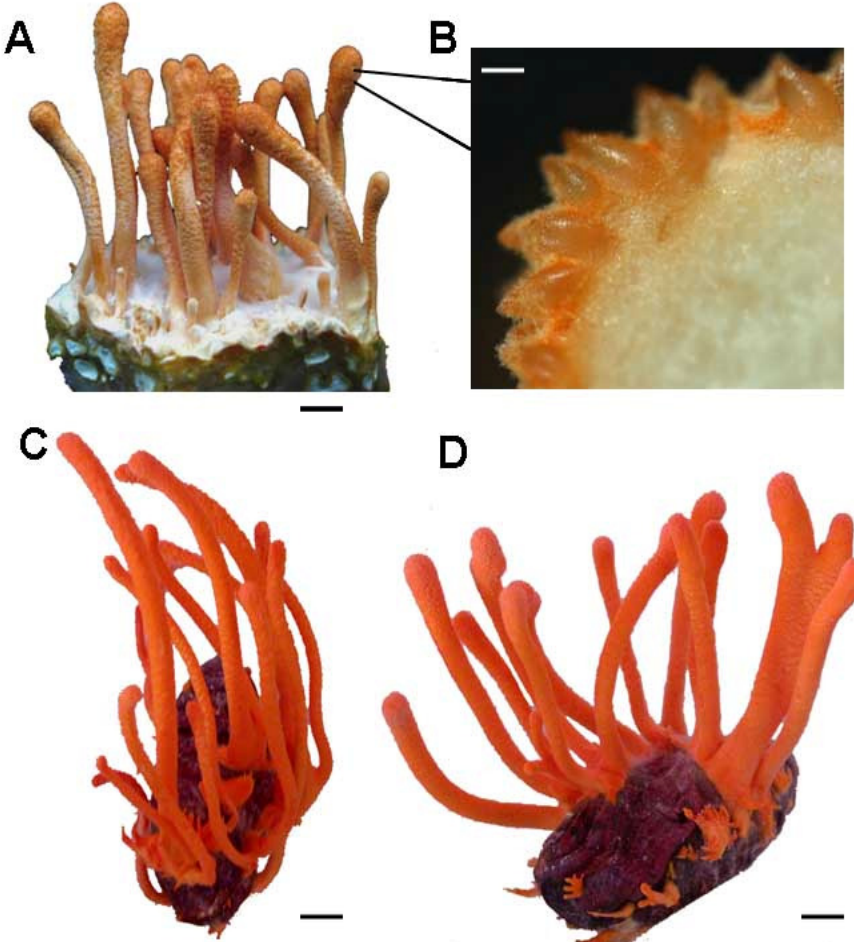

D

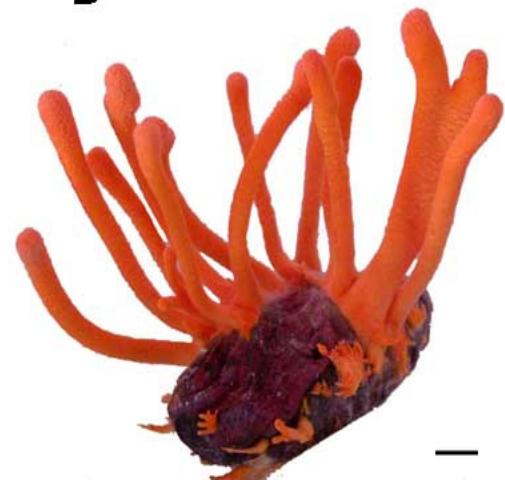

Figure 2. Sexual reproduction of C. cicadae MP12. A: Fruiting bodies growing on the RSM medium; B: The mature stroma from the fruiting body growing on the RSM medium; C and D: C. cicadae MP12 on cicadae pupae after inoculation. Scale bars: A, C-D = $10 \mathrm{~mm}$; $\mathrm{B}=100 \mu \mathrm{m}$.

\section{Morphological characters and molecular data of $\boldsymbol{C}$. cicadae} MP12

In the conidial stage of $C$. cicadae MP12, displayed an abundance of branched septate mycelium and the conidiospore started germinating after 7 days at $25^{\circ} \mathrm{C}$ on Sabouraud dextrose agar (Fig 1A). The aerial mycelium is cottony in texture and whitish yellow-colored in the middle, the reverse side of cultures is yellowish. The microscopic observation showed that conidiospores were produced in stringed chains. Stromata was observed solitary or occasionally with serveral, simple or rarely branched, on the larva of cicada and RSA medium. Stipe was fleshly, ochraceous orange to red, clavate, 5-50×0.5-6 mm. Fertile area on the terminal was reddish orange to reddish, cylindrical, elliptical to fusiform, 3-10×1-6 mm. Perithecia were crowded, loosely embedded, ordinal-likely in orientation, elliptical to fusiform to obclavate, $200-600 \times 150-250 \mu \mathrm{m}$. The typical orange bat-shaped stromata were fruiting from cicadae and RSM medium 14 days after inoculation using conidiospores. The oval-shaped perithecia were presented on the stroma with cream-colored prosenchyma inside (Fig 2 B).

After SSU rDNA amplification, a 1150 bp DNA fragment was obtained and sequenced (NCBI access number HM536623). Final alignments for phylogenetic analysis included characters from various species of Clavicipitaceae used in Philip alignments. Parsimony analysis showed that $C$. cicadae MP12 was closer to another C. cicada (NCBI access number DQ838788) and C. inegoensis phylogenetically (Fig. 3). The topology of the most-parsimonious tree and the Cordyceps phylogenetic pattern were globally similar to the results of a larger phylogenetic analysis of the Clavicipitaceae with more intensive taxon sampling (21).

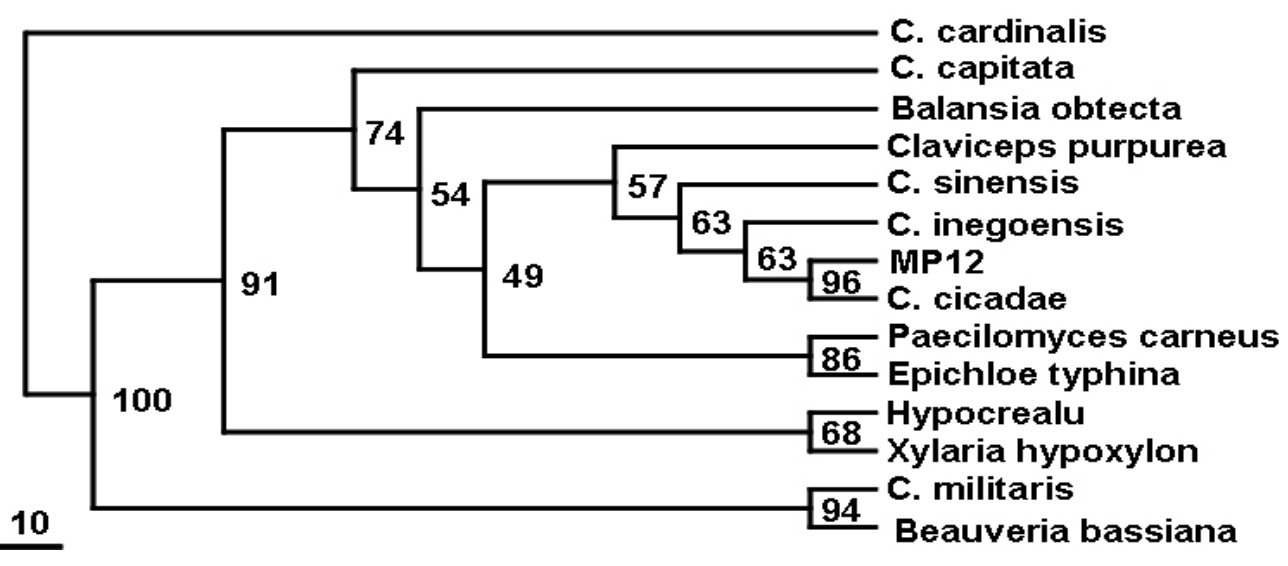

Figure 3. The most-parsimonious tree from the maximum parsimony analysis of SSU rDNA data. Numbers above nodes are nonparametric bootstrap values from 1000 replications. MP12 indicates C. cicadae MP12. 
Production of adenosine and cordycepin in $C$. cicadae MP12

The peaks of adenosine and cordycepin were identified at the Retention Time of $10.78 \mathrm{~min}$ and $14.24 \mathrm{~min}$, repectively (Fig. 4A). Two peaks corresponding to adenosine and cordycepin from the extract of dried mycelium (10.92 min and $14.41 \mathrm{~min}$ ) and fruiting bodies from in vitro culture (10.89 $\mathrm{min}$ and $14.35 \mathrm{~min}$ ) and in vivo culture (11.01 $\mathrm{min}$ and $14.43 \mathrm{~min}$ ) were detected and after a peak correction $(p<0.05)$ (Fig. 4B, C, D). Standard adenosine concentrations ranging from 50 to 500 $\mu \mathrm{g} / \mathrm{ml}$ were used to establish the linear curve between concentration and peak area. The equation that $\mathrm{Y}=62.75 \mathrm{X}$ 550.73 (Y, concentration, $\mu \mathrm{g} / \mathrm{ml}$; $\mathrm{X}$, peak area, $\mathrm{mU} \times \min$ ) was established after three replicates $(p<0.05)$. With the same procedure, standard cordycepin concentrations ranging from 50 to $500 \mu \mathrm{g} / \mathrm{ml}$ came to the equation $\mathrm{Y}=34.14 \mathrm{X}-443.50$ ( $\mathrm{Y}$, concentration, $\mu \mathrm{g} / \mathrm{ml}$; $\mathrm{X}$, peak area, $\mathrm{mU} \times \min )(p<0.05)$ in the chromatograms. According to these two standard models, the content of adenosine in the dried mycelium was $82.39 \mu \mathrm{g} / \mathrm{g}$, much lower than the extract of dried fruiting body cultured in RSM medium (1398.12 $\mu \mathrm{g} / \mathrm{g})$ and dried fruiting body growing on cicada $(1421.45 \mu \mathrm{g} / \mathrm{g})$. Also, the contents of cordycepin were $64.98 \mu \mathrm{g} / \mathrm{g}$ in the mycelium, $1548.12 \mu \mathrm{g} / \mathrm{g}$ in the dried fruiting body cultured in the RSM medium, and $1578.43 \mu \mathrm{g} / \mathrm{g}$ in the dried fruiting body growing in the cicada larvae. The contents of adenosine and cordycepin in the fruiting body showed no significant difference between culturing in RSM medium and harvest on cicada $(p<0.05)$.
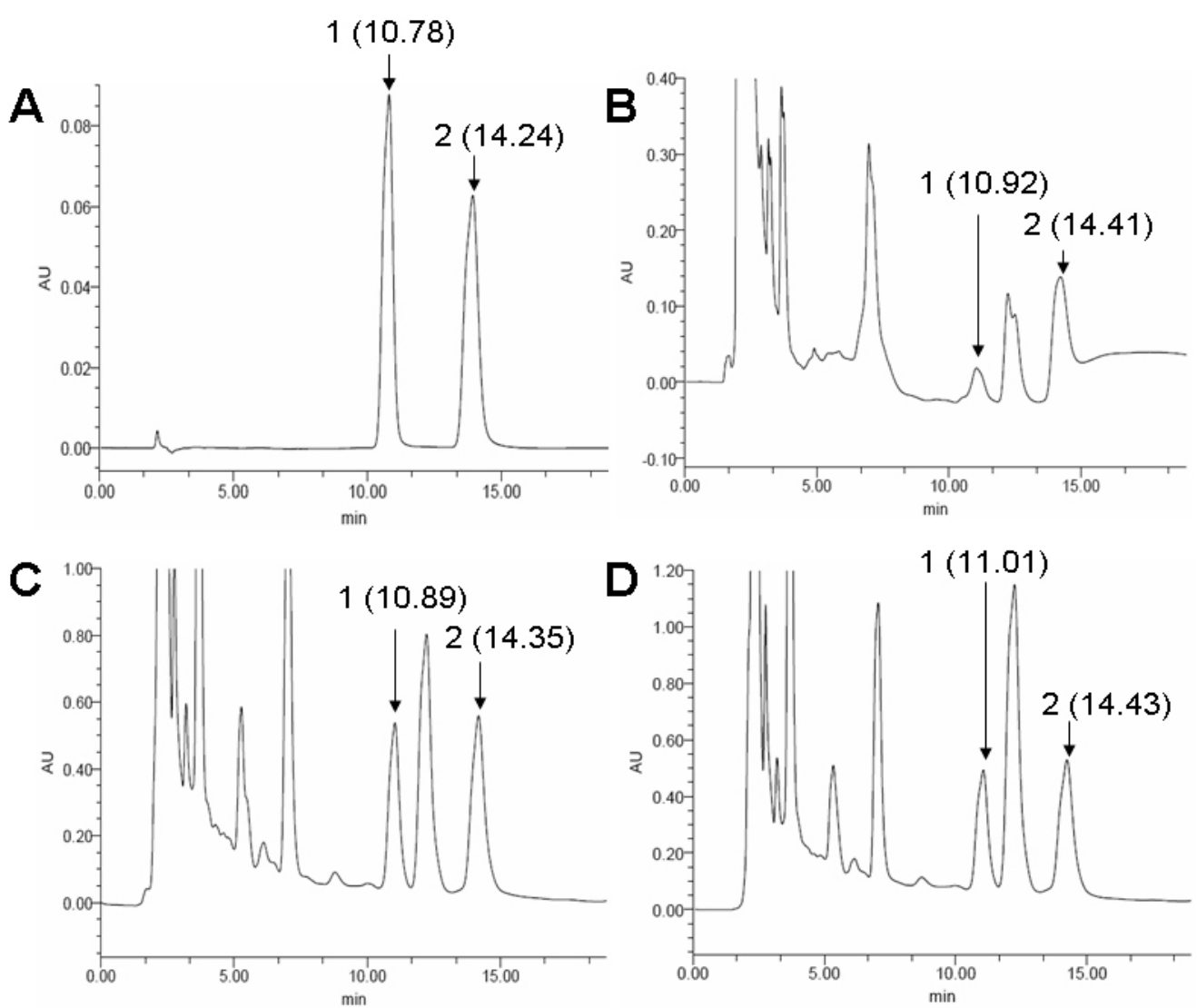

Figure 4. Chromatograms (HPLC) of water solution extracted from C. cicadae MP12. A: Standard adenosine (80 $\mu \mathrm{g} / \mathrm{ml})$ and cordycepin $(100 \mu \mathrm{g} / \mathrm{ml})$; B: water extract of dried mycelium; C: water extract of the harvested fruiting body of C. cicadae MP12 cultured in the RSM medium; D: water extract of harvested fruiting body of C. cicadae MP12 growing on cicadae pupae of after inoculation. Peak 1 and peak 2 with retention time followed in the four panes indicate adenosine and cordycepin, respectively. 


\section{DISCUSSION}

Cordyceps (Fr.) includes pathogens of species from nine orders of arthropods and parasites of one genus, Elaphomyces. In the Cordyceps genus, $C$. militaris is one of the most frequently collected species. However, it is also one of the most variable species in the genus with respect to morphology and host affiliation (21). Sung et al. conducted the most extensive multigene phylogenetic analyses to provide a basis for the phylogenetic classification of Cordyceps and the clavicipitaceous fungi, and determined the clear classification of this genus (21). Also, based on the evolution of host specificity, the phylogenetic relationships and the stromata morphological characters, five monophyletic groups in Cordyceps genus were identified: the truffle-cicada clade, cicada clade A, cicada clade B, the scale-moth clade, and the moth clade (17). Previously, C. cicadae MP12 was also considered non-specific with $C$. militaris according to its macroscopical morphology. In the present study, inoculation of C. cicadae MP12 on cicadae and morphological characters, including clavate stroma, oval crowed perithecia, branched mycelium and conidiospores producing in stringed chains, grouped this isolate into the cicada clade $\mathrm{A}$, which includes $C$. inegoensis and C. paradoxa. The high similarity of SSU rDNA sequences between $C$. inegoensis and $C$. cicadae MP12 is consistent with this group. The anamorphic and cultural differences from $C$. inegoensis suggested that isolate MP12 was more similar to $C$. cicadae.

Various bioactive constituents from Cordyceps species have been reported. These include cordycepin and other antibacterial and anti-tumour adenosine derivatives, ophicordin, an anti-fungal agent, myriocin, L-tryptophan, etc $(16,26)$. In this study, the contents of adenosine and cordycepin were measured by the use of HPLC. In the chromatographic data, peaks corresponding to standard adenosine and cordycepin were identified. These results indicated that the contents of both adenosine and cordycepin in C. cicadae MP12 were about the same as those in $C$. militaris $(13,24,25)$.

The fruiting body culture on a large scale using in vitro media has recently been developed to produce cordycepin due to its limited supply from natural sources. Therefore, several methods have been established for cultivation of varied Cordyceps species $(10,12,14,23)$. In this case, an economical and high efficient cultivation for C. cicadae in vitro and in vivo was first-time established to produce the fruiting body. This culturing would help to identify other pharmacologically active metabolites from C. cicadae in the future.

\section{ACKNOWLEDGEMENTS}

This work was supported by a grant from Qianjiang Talents Grant, No. 2010R10098 funded by the Department of Science \& Technology of Zhejiang Province, China and by a grant from National Natural Science Foundation of China (NSFC 30901153). We are thankful to Dr. Anliang Chen for the assistance on HPLC work.

\section{REFERENCES}

1. Berne, R.M. (1980). The role of adenosine in the regulation of coronary blood flow. Circ. Res. 47, 807-813.

2. Cunningham, K.G.; Manson, W.; Spring, F.S.; Hutchinson, S.A. (1950). Cordycepin, a metabolic product isolated from cultures of Cordyceps militaris (Linn.) Link. Nature 166, 949.

3. Felsenstein, J. (1988). Phylogenies from molecular sequences: inference and reliability. Annu. Rev. Genet. 22, 521-565.

4. Hermann, S.C.; Feigl, E.O. (1992). Adrenergic blockade blunts adenosine concentration and coronary vasodilation during hypoxia. Circ. Res. 70, 1203-1216.

5. Holliday, J.; Cleaver, M. (2008). Medicinal Value of the Caterpillar Fungi Species of the Genus Cordyceps (Fr.) Link (Ascomycetes). A Review. Int. J. Med. Mushrooms 10, 219-234.

6. Ioannidis, P.; Courtis, N.; Havredaki, M.; Michailakis, E.; Tsiapalis, C.M.; Trangas, T. (1999). The polyadenylation inhibitor cordycepin (3'dA) causes a decline in c-MYC mRNA levels without a.ecting cMYC protein levels. Oncogene 18, 117-125.

7. Kaczka, E.A.; Trenner, N.R.; Arison, B.; Walker, R.W.; Folkers, K. (1964). Identification of cordycepin, a metabolite of Cordyceps militaris, 
as 3'-deoxyadenosine. Biochem. Biophys. Res. Commun. 14, 456-457.

8. Kodama, E.N.; McCaffrey, R.P,; Yusa, K.; Mitsuya, H. (2000). Antileukemic activity and mechanism of action of cordycepin against terminal deoxynucleotidyl transferase-positive $(\mathrm{TdT}+)$ leukemic cells. Biochem. Pharmacol. 59, 273-281.

9. Kuo, Y.C.; Weng, S.C.; Chou, C.J.; Chang, T.T.; Tsai, W.J. (2003). Activation and proliferation signals in primary human $\mathrm{T}$ lymphocytes inhibited by ergosterol peroxide isolated from Cordyceps cicadae. Br. J. Pharmacol. 140, 895-906.

10. Lee, S.Y.; Nakajima, I.; Ihara, F.; Kinoshita, H.; Nihira, T. (2005) Cultivation of entomopathogenic fungi for the search of antibacterial compounds. Mycopathologia 160, 321-325.

11. Legraverend, M.; Robert, I. (1978). Glazer inhibition of the phosphorylation of non-histone chromosomal proteins of rat liver by cordycepin and cordycepin triphosphate. Cancer Res. 38, 1142-1146.

12. Lin, Q.Y.; Song, B.; Huang, H.; Li, T.H. (2010). Optimization of selected cultivation parameters for Cordyceps guangdongensis. Lett. Appl. Microbiol. 51, 219-225.

13. Ling, J.Y.; Sun, Y.J.; Zhang, H.; Lv, P.; Zhang, C.K. (2002). Measurement of cordycepin and adenosine in stroma of Cordyceps sp. by capillary zone electrophoresis (CZE). J. Biosci. Bioeng. 94, 371-374.

14. Mao, X.B.; Zhong, J.J. (2004). Hyperproduction of cordycepin by twostage dissolved oxygen control in submerged cultivation of medicinal mushroom Cordyceps militaris in bioreactors. Biotechnol. Prog. 20, 1408-1413.

15. Müller, W.E.G.; Seibert, G.; Beyer, R.; Breter, H.J.; Maidhof, A.; Zahn, R.K. (1977). Effect of cordycepin on nucleic acid metabolism in L5178Y cells and on nucleic acid-synthesizing enzyme systems. Cancer Res. 37, 3824-3833.

16. Ng, T.B.; Wang, H.X. (2005). Pharmacological actions of Cordyceps, a prized folk medicine. J. Pharm. Pharmacol. 57, 1509-1519.

17. Nikoh, N.; Fukatsu, T. (2000). Interkingdom host jumping underground: Phylogenetic analysis of entomoparasitic fungi of the genus Cordyceps. Mol. Biol. Evol. 17, 629-638.

18. Pelleg, A.; Porter, R.S. (1990). The pharmacology of adenosine.
Pharmacotherapy 10, 157-174.

19. Russell, R.; Paterson, M. (2008). Cordyceps - A traditional Chinese medicine and another fungal therapeutic biofactory? Phytochemistry 69, 1469-1495.

20. Shin, S.; Lee, S.; Kwon, J.; Moon, S.; Lee, S.; Lee, C-K.; Cho, K.; Ha, N-J.; Kim, K. (2009). Cordycepin suppresses expression of diabetes regulating genes by inhibition of lipopolysaccharide-induced inflammation in macrophages. Immune. Netw. 9, 98-105.

21. Sung, G-H.; Hywel-Jones, N.L.; Sung, J-M.; Luangsa-Ard, J.J.; Shrestha, B.; Spatafora, J.W. (2007). Phylogenetic classification of Cordyceps and the clavicipitaceous fungi. Stud. Mycol. 57, 5-59.

22. Wong, Y.Y.; Moon, A.; Duffin, R.; Barthet-Barateig, A.; Meijer, H.A.; Clemens, M.J.; de Moor, C.H. (2010). Cordycepin inhibits protein systhesis and cell adhesion through effects on signal transduction. $J$. Biol. Chem. 285, 2610-2621.

23. Wongsa, P.; Tasanatai, K.; Watts, P.; Hywel-Jones, N. (2005). Isolation and in vitro cultivation of the insect pathogenic fungus Cordyceps unilateralis. Mycol. Res. 109, 936-940.

24. Xie, C.Y.; Gu, Z.X.; Fan, G.J.; Gu, F.R.; Han, Y.B.; Chen, Z.G. (2009). Production of cordycepin and mycelia by submerged fermentation of Cordyceps militaris in mixture natural culture. Appl. Biochem. Biotechnol. 158, 483-492.

25. Yang, F.Q.; Li, D.Q.; Feng, K.; Hu, D.J.; Li, S.P. (2010). Determination of nucleotides, nucleosides and their transformation products in Cordyceps by ion-pairing reversed-phase liquid chromatography-mass spectrometry. J. Chromatogr. A. 1217, 5501-5510.

26. Yu. J.; Xu, H.; Mo, Z.; Zhu, H.; Mao, X. (2009). Determination of myriocin in natural and cultured Cordyceps cicadae using 9fluorenylmethyl chloroformate derivatization and high-performance liquid chromatography with UV-detection. Anal. Sci. 25, 855-859.

27. Yu, R.; Yang, W.; Song, L.; Yan, C.; Zhang, Z.; Zhao, Y. (2007). Structural characterization and antioxidant activity of a polysaccharide from the fruiting bodies of cultured Cordyceps militaris. Carbohydr. Polym. 70, 430-436. 